\title{
Clinical value of black-blood high-resolution magnetic resonance imaging for intracranial atherosclerotic plaques
}

\author{
HAIQING YANG ${ }^{1}$, YIFEI ZHU ${ }^{2}$, ZUOJUN GENG $^{1}$, CAIYING LI $^{1}$, LIXIA ZHOU $^{1}$ and QI LIU ${ }^{2}$ \\ Departments of ${ }^{1}$ Radiology and ${ }^{2}$ Neurology, The Second Hospital of Hebei Medical University, \\ Shijiazhuang, Hebei 050000, P.R. China
}

Received July 8, 2014; Accepted March 20, 2015

DOI: $10.3892 /$ etm.2015.2469

\begin{abstract}
The present study evaluated the value of black-blood high-resolution magnetic resonance imaging (HRMRI) for the visualization of intracranial atherosclerosis (ICAS) plaques. A total of 110 patients with cerebral artery or vertebrobasilar stenosis, vessel occlusion or a significantly weakened signal in black-blood magnetic resonance angiography (MRA; three-dimensional time-of-flight) were examined. Black-blood MRA was used to observe whether plaques were present in the abnormal vascular walls. Among the 110 patients with cerebral infarction, 16 cases presented with no significant abnormality of the lumen and walls, while plaques were observed in 94 cases. The plaques were categorized according to their signal characteristics, which resulted in the identification of four cases of type I and II plaques, 15 cases of type III, 26 cases of type IV and V, 23 cases of type VI, 11 cases of type VII, 14 cases of type VIII and one case of a mixed plaque. In summary, 3.0 T black-blood HRMRI was demonstrated to objectively exhibit characteristics of various types of ICAS plaques. Therefore, this imaging technique may be applied as a key method for the clinical non-invasive determination of ICAS plaques.
\end{abstract}

\section{Introduction}

Intracranial atherosclerosis (ICAS) is the most common cause of ischemic stroke worldwide. To date, the incidence of ICAS-induced stroke in China has exceeded those in other countries and is the primary cause of mortality and disability in adult patients (1). The risk of stroke is associated with the degree of artery stenosis and the stability of atherosclerotic plaques. However, only a small proportion of stroke cases are a result of intracranial arterial stenosis only. Magnetic

Correspondence to: Dr Yifei Zhu, Department of Neurology, The Second Hospital of Hebei Medical University, 215 Heping West Road, Shijiazhuang, Hebei 050000, P.R. China

E-mail: yifeizhucn@163.com

Key words: magnetic resonance imaging, intracranial atherosclerosis, black-blood, bright-blood resonance angiography (MRA) technology is able to visualize cerebral arterial plaque composition, activity characteristics and morphological alterations of blood vessels, in addition to the corresponding changes of cerebral artery plaques in cerebral infarction patients following clinical drug therapy. Cerebral infarction may be caused by a number of factors, including ICAS plaque rupture, hemorrhage, thrombogenesis and plaque detachment $(2,3)$. Thus, the early determination of ICAS, particularly the early determination of plaques and the vulnerability of plaques, is a key method for reducing the incidence rate of cerebral infarction. In the present study, qualitative analysis of ICAS plaques was conducted using 3.0 T black-blood high-resolution magnetic resonance imaging (HRMRI), with the aim to assess the clinical value of black-blood MRA and the prevention of ICAS plaque formation.

\section{Subjects and methods}

Subjects. A total of 110 patients with cerebral infarction were recruited from the outpatient and inpatient departments of the Second Hospital of Hebei Medical University (Hebei, China) between the $1^{\text {st }}$ January 2012 and $1^{\text {st }}$ December 2013. The study population consisted of 63 male and 47 female patients, with an average age of $58.6 \pm 5$ years (range, $30-80$ years). Three-dimensional time-of-flight (3D-TOF) MRA, also known as bright-blood technology, presented cerebral artery stenosis, local vertebrobasilar stenosis or signal interruptions. Subsequently, black-blood MRA was used to scan the abnormal blood vessels and to observe whether plaques existed in the vessel walls. The MRI signal characteristics of the plaques were observed and categorized. The study was conducted in accordance with the Declaration of Helsinki and with approval from the Ethics Committee of the Second Hospital of Hebei Medical University. Written informed consent was obtained from all the participants.

MRI examinations. A Signa Excite HD 3.0 T MRI scanner (GE Healthcare Life Sciences, Bethesda, MD, USA) was employed in the study for all imaging techniques, using the standard eight-channel orthogonal array coil. For the black-blood MRA examinations, double inversion recovery fast spin echo (DIR-FSE) T1-weighted imaging (T1WI; fat-suppressed) was performed with a repetition time (TR) 
Table I. Plaque classification, signal characteristics of black-blood MRA and vascular wall condition.

MRI signal characteristics

\begin{tabular}{|c|c|c|c|c|c|c|}
\hline \multirow{2}{*}{$\begin{array}{l}\text { Plaque } \\
\text { type }\end{array}$} & \multirow[b]{2}{*}{ Cases (n) } & \multirow[b]{2}{*}{ Ratio (\%) } & \multirow{2}{*}{$\begin{array}{c}\text { Wall } \\
\text { characteristics }\end{array}$} & \\
\hline & & & & T1WI (fat saturation) & $\mathrm{T} 2 \mathrm{WI}$ & PDW \\
\hline I,II & 4 & 4.3 & $\begin{array}{l}\text { No significant } \\
\text { thickening }\end{array}$ & Equisignal & Equisignal & Equisignal \\
\hline III & 15 & 16.0 & $\begin{array}{l}\text { Nonuniform } \\
\text { thickening and } \\
\text { small plaque }\end{array}$ & $\begin{array}{l}\text { Comparatively } \\
\text { uniform } \\
\text { equisignal }\end{array}$ & $\begin{array}{l}\text { Comparatively } \\
\text { uniform and } \\
\text { high signal }\end{array}$ & $\begin{array}{l}\text { Comparatively } \\
\text { uniform and } \\
\text { high signal }\end{array}$ \\
\hline IV,V & 26 & 27.7 & $\begin{array}{l}\text { Nonuniform } \\
\text { thickening and } \\
\text { eccentric plaque }\end{array}$ & $\begin{array}{l}\text { Equisignal and } \\
\text { comparatively } \\
\text { low signal }\end{array}$ & $\begin{array}{l}\text { Equisignal and } \\
\text { high signal }\end{array}$ & $\begin{array}{l}\text { Equisignal and } \\
\text { high signal }\end{array}$ \\
\hline VI & 23 & 24.5 & $\begin{array}{l}\text { Eccentric } \\
\text { plaque }\end{array}$ & $\begin{array}{l}\text { Equisignal and } \\
\text { comparatively } \\
\text { high signal }\end{array}$ & $\begin{array}{l}\text { Equisignal and } \\
\text { comparatively } \\
\text { high signal }\end{array}$ & $\begin{array}{l}\text { Equisignal and } \\
\text { comparatively } \\
\text { high signal }\end{array}$ \\
\hline VII & 11 & 11.7 & $\begin{array}{l}\text { Eccentric } \\
\text { plaque }\end{array}$ & Low signal & Low signal & Low signal \\
\hline VIII & 14 & 14.9 & $\begin{array}{l}\text { Nonuniform } \\
\text { thickening and } \\
\text { eccentric plaque }\end{array}$ & $\begin{array}{l}\text { Equisignal and } \\
\text { low signal }\end{array}$ & $\begin{array}{l}\text { Equisignal and } \\
\text { low signal }\end{array}$ & $\begin{array}{l}\text { Equisignal and } \\
\text { low signal }\end{array}$ \\
\hline $\begin{array}{l}\text { Mixed } \\
\text { plaque }\end{array}$ & 1 & 1.1 & $\begin{array}{l}\text { Irregular } \\
\text { concentric ring } \\
\text { plaque }\end{array}$ & $\begin{array}{l}\text { Equisignal and } \\
\text { low signal }\end{array}$ & $\begin{array}{l}\text { Mixture of high, } \\
\text { equisignal and } \\
\text { low signals }\end{array}$ & $\begin{array}{l}\text { Mixture of high, } \\
\text { equisignal and } \\
\text { low signals }\end{array}$ \\
\hline
\end{tabular}

MRI, magnetic resonance imaging; T1WI, T1-weighted imaging; T2WI, T2-weighted imaging; PDW, proton density-weighted.

of $800 \mathrm{msec}$, an inversion time (TI) of $300 \mathrm{msec}$ and an echo time (TE) set at the minimum time at which a full echo was obtainable (min full). DIR-FSE proton density-weighted imaging (PDWI) was performed with a TR of 3,000 msec and a TE of min full. Furthermore, DIR-FSE T2-weighted imaging (T2WI) was conducted with a TR of 3,000 msec and a TE of $102 \mathrm{msec}$. The field of vision was 120x120 mm and the array was $512 \times 512$, with a number of excitation (NEX) of three. For the bright-blood imaging, the 3D-TOF scanning method was applied with a TR of $29 \mathrm{msec}$ and a TE of $2.1 \mathrm{msec}$.

Image analysis. In accordance with the revised American Heart Association Pathological and Histological Classification (4), two senior neuroradiologists evaluated the plaques in the lesion areas of the M1 section of the middle cerebral artery and basilar artery using MRI classification criteria, as described by Cai et al (2). The plaque characteristics, including the type and vulnerability, were subsequently analyzed. The plaque signal level was determined using the level in the adjacent brain parenchyma as the reference, and was characterized as a high signal, equisignal or low signal. The main components in the analysis included the presence of a lipid core, calcification, hemorrhage and fibrous caps.

Statistical analysis. Statistical analysis was performed using SPSS software, version 15.0 (SPSS Inc., Chicago, IL, USA). Categorical variables were tested using the $\chi^{2}$ test. $\mathrm{P}<0.05$ was considered to indicate a statistically significant difference.

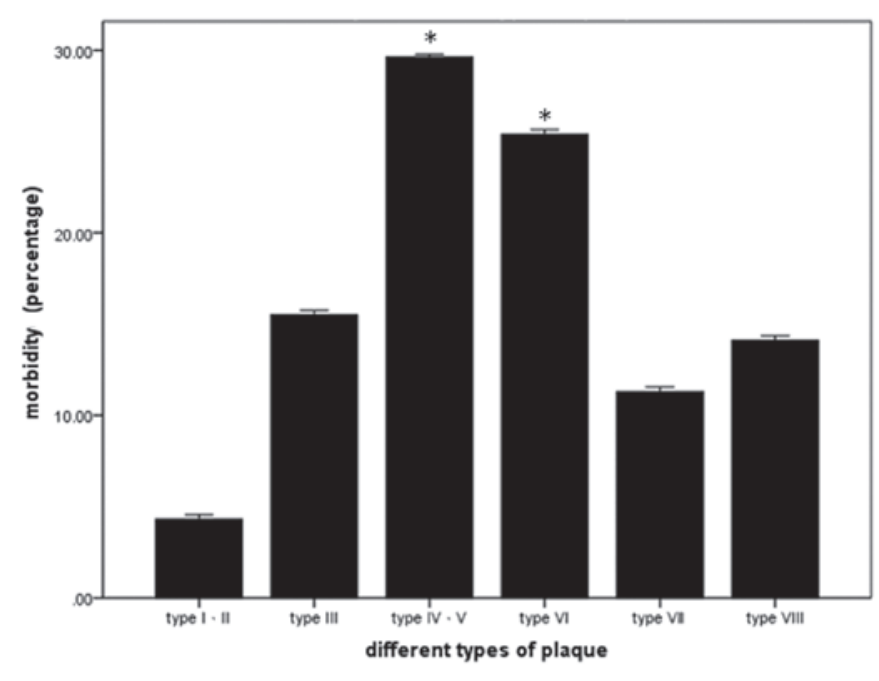

Figure 1 . Morbidity rates of the different plaque types. ${ }^{*} \mathrm{P}<0.05$ compared with other plaque types.

\section{Results}

Detection of plaques. Bright-blood 3D-TOF magnetic resonance angiography (MRA) identified 110 cases with abnormal cerebral vessels. However, black-blood MRA identified 16 cases $(14.5 \%)$ without an abnormality or plaque in the cerebral artery lumen or walls, and 94 cases $(85.5 \%)$ with various types of cerebral artery plaques, as well as lumen stenosis at varying degrees. 

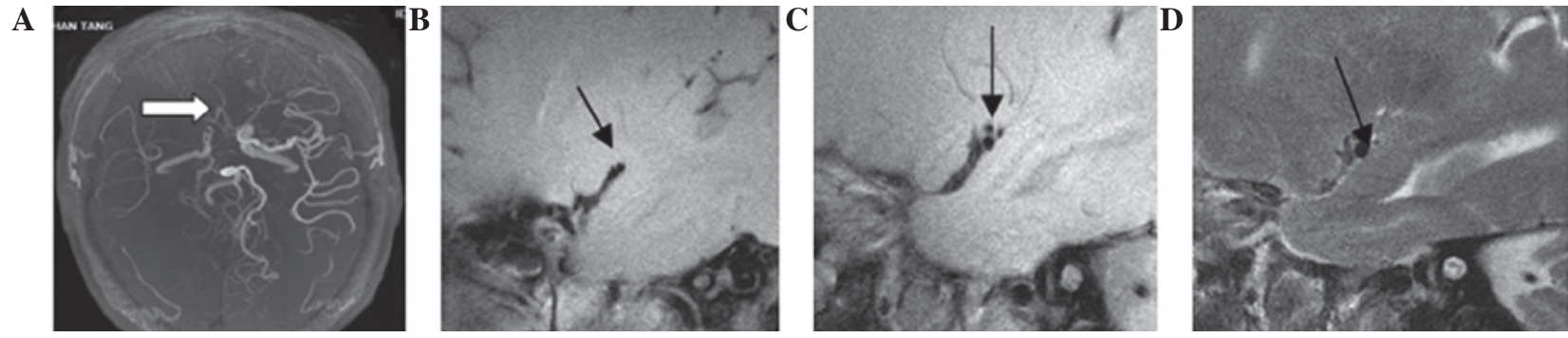

Figure 2. Plaque types I and II. (A) 3D-time-of-flight magnetic resonance angiography indicated that the right cerebral arterial signal had disappeared. Images of black-blood magnetic resonance (B) T1-weighted imaging (fat-suppressed), (C) proton density-weighted imaging and (D) T2-weighted imaging, which clearly demonstrate the existence of a lumen and enhanced wall thickness.

A

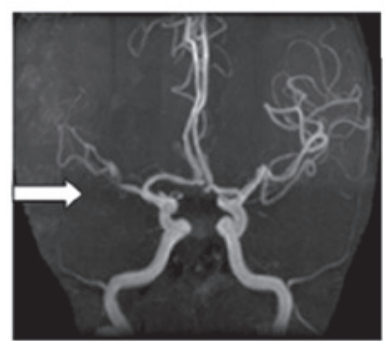

B

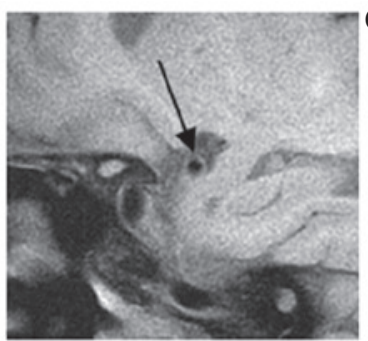

C

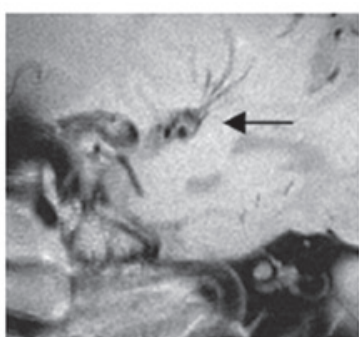

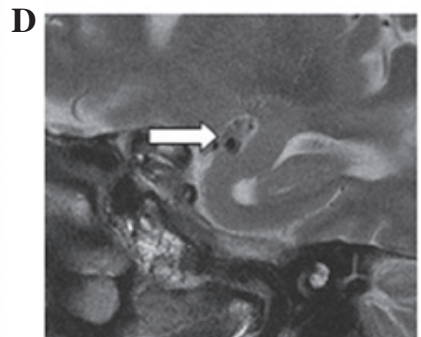

Figure 3. Plaque type III. (A) 3D-time-of-flight magnetic resonance angiography of right cerebral arterial local lumen stenosis. Images of black-blood magnetic resonance (B) T1-weighted imaging (fat-suppressed), (C) proton density-weighted imaging and (D) T2-weighted imaging, which clearly present cerebral artery stenosis and the equisignal of walls at irregular thicknesses.

A

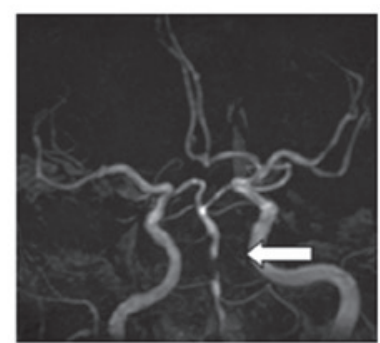

B

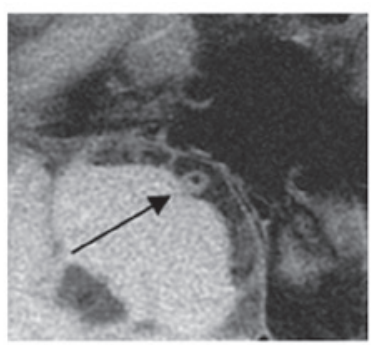

$\mathrm{C}$

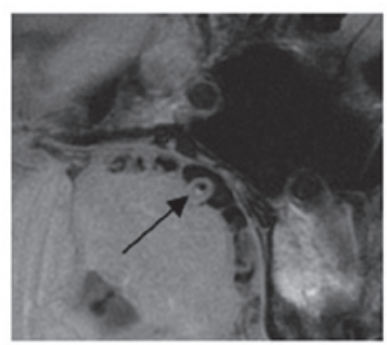

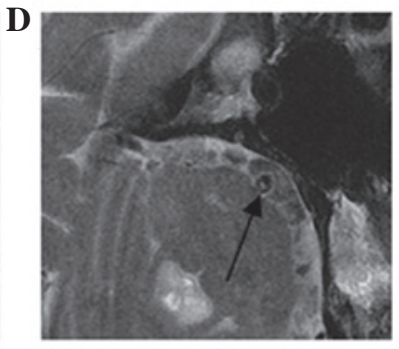

Figure 4. Plaque types IV and V. (A) 3D-time-of-flight magnetic resonance angiography of multistage stenosis of the basilar artery. (B) Plaque shows an equisignal in the black-blood magnetic resonance T1-weighted imaging (fat-suppressed). Strips of high signal were observed in the equisignal plaques in (C) proton density-weighted imaging and (D) T2-weighted imaging.
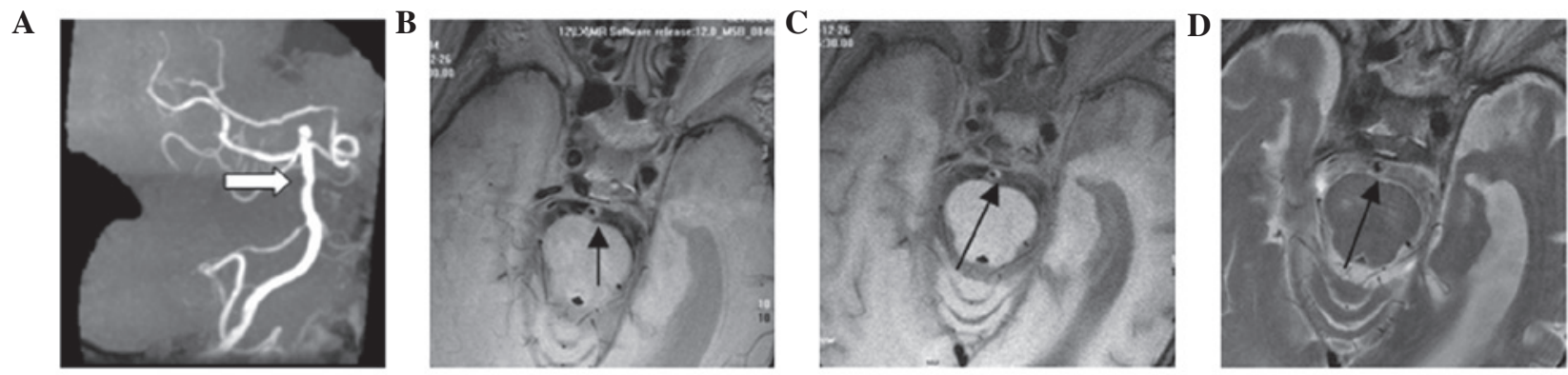

Figure 5. Plaque type VI. (A) 3D-time-of-flight magnetic resonance angiography revealed local stenosis of the basilar artery. Images from black-blood magnetic resonance (B) T1-weighted imaging (fat-suppressed), (C) proton density-weighted imaging and (D) T2-weighted imaging, displaying plates of high signal (bleeds) in an equisignal plaque of the basilar artery lumen.

Classification of plaques. According to the MRI signal characteristics and classification criteria of the plaques, an atherosclerotic plaque was detected in 94 cases, which included four cases of types I and II, 15 cases of type III, 26 cases of type IV and V, 23 cases of type VI, 11 cases of type VII,
14 cases of type VIII and one case of a mixed/complex plaque (Table I).

Incidence of plaque types. Statistical analysis indicated that the incidence of type IV, V and VI atherosclerotic plaques 
A

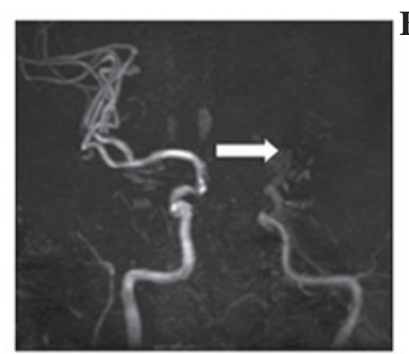

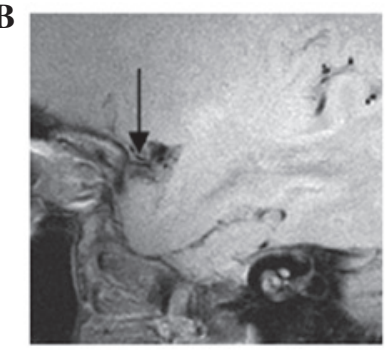

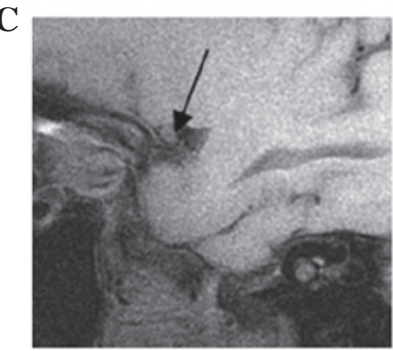

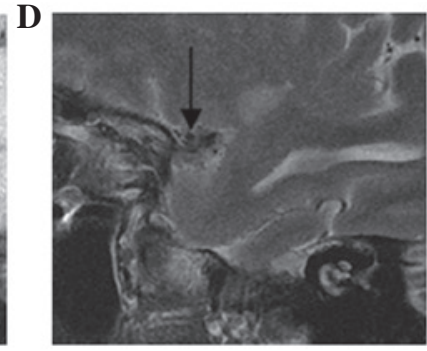

Figure 6. Plaque type VII. (A) 3D-time-of-flight magnetic resonance angiography presented no development of the left cerebral artery. Equisignal plaque images were observed in the black-blood magnetic resonance (B) T1-weighted imaging (fat-suppressed), (C) proton density-weighted imaging and (D) T2-weighted imaging.
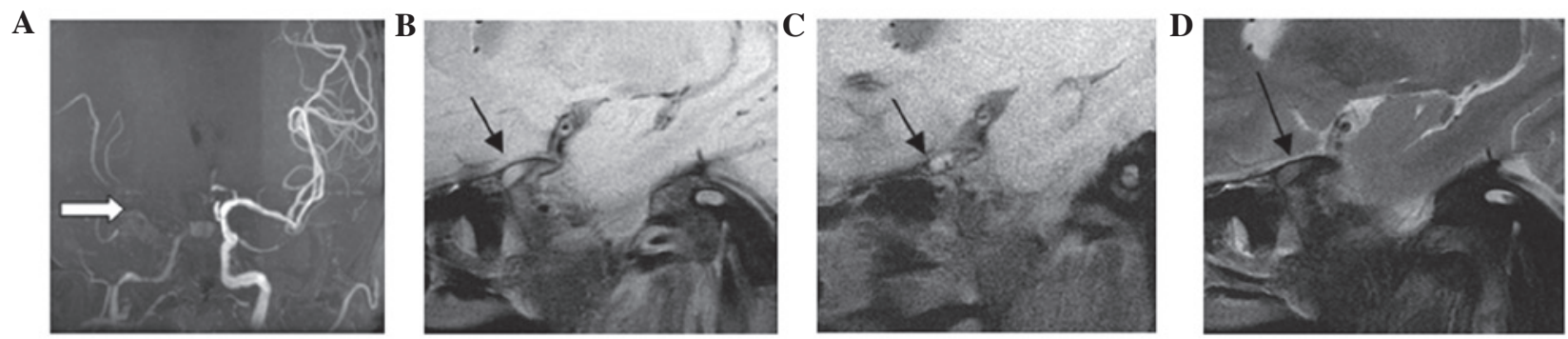

Figure 7. Plaque type VIII. (A) 3D-time-of-flight magnetic resonance angiography presented no development of the right cerebral artery M1. Equisignal plaque images were observed in the black-blood magnetic resonance (B) T1-weighted imaging (fat-suppressed), (C) proton density-weighted imaging and (D) T2-weighted imaging.
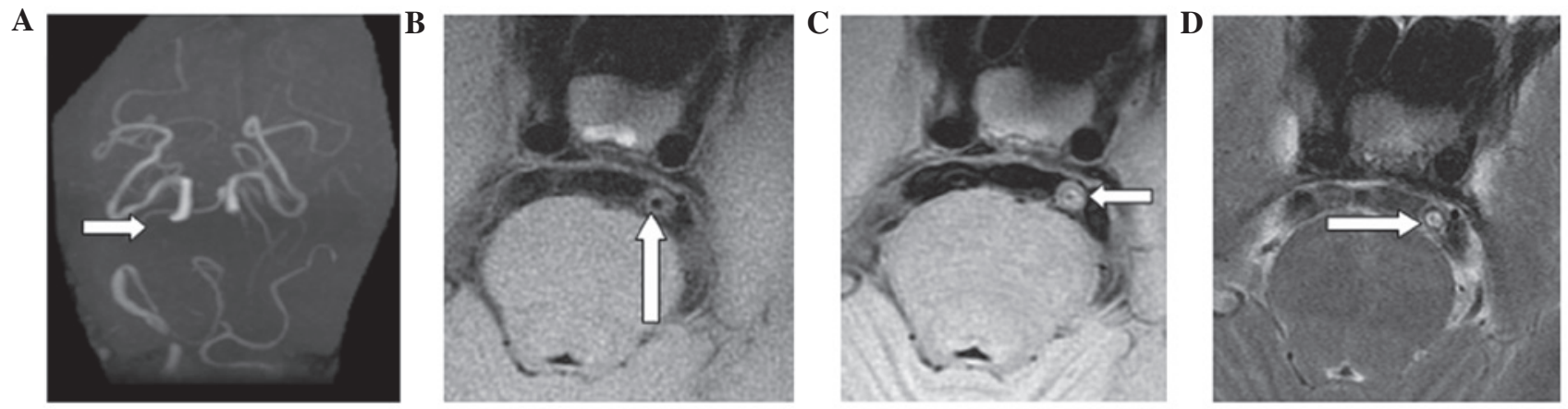

Figure 8. Mixed plaque type. (A) 3D-time-of-flight magnetic resonance angiography presented basilar artery stage stenosis, or even occlusion. Complex signals (high, equisignal and low) were observed in the plaques from the black-blood magnetic resonance (B) T1-weighted imaging (fat-suppressed), (C) proton density-weighted imaging and (D) T2-weighted imaging plaque.

was significantly higher compared with other types of plaque (Fig. 1).

\section{Discussion}

As quality of life improves and the population subsequently ages, ICAS-induced cerebrovascular diseases have become increasingly prevalent in clinical practice. Various mechanisms underlying cerebrovascular diseases caused by intracranial artery lesions have been proposed, including a reduced cerebral blood flow due to cerebral artery stenosis and an arterial embolism caused by ICAS due to plaque rupture and detachment, thus resulting in transient ischemic attack and stroke $(5,6)$. ICAS plaque vulnerability has presented increased risk of cerebral infarction compared with hemadostenosis (7). Plaque stability is primarily determined by the size of the lipid core, the thickness of the fibrous cap, and the extent of hemorrhage, inflammatory reaction or new vessel formation. Additionally, a histopathological study by Cappendijk et al (8) demonstrated that a large, necrotic lipid core is the most important factor affecting plaque vulnerability. Thus, early determination of plaque vulnerability is a key approach for the prevention of plaque detachment and subsequent cerebral infarction.

Various traditional methods of intracranial artery examination exist, including transcranial Doppler ultrasound (TCD), bright-blood 3D-TOF MRA, computed tomography angiography (CTA) and digital subtraction angiography (DSA). TCD has limited use in cerebral artery examination due to the position of the cranium. A number of studies have used TCD in cerebral artery examination; however, these studies observed the extent of stenosis and blood flow velocity in the cerebral artery, but did not conduct a detailed examination of the plaques. Thus, the plaque type and stability were unable to be determined. Cerebral artery CTA focuses on identifying 
the existence and size of arterial aneurysms, in addition to the degree of stenosis. CTA permits approximate observation of calcified, soft and mixed plaques. DSA is the gold standard for determining the degree of cerebrovascular stenosis and is applicable to invasive examinations. However, DSA requires the administration of a significant dose of radiation, and the DSA method can result in embolus detachment, which may inadvertently cause cerebral infarction. Therefore, the clinical applications of DSA are limited (9).

MRA possesses a number of advantages, including non-invasiveness, no requirement for radiation, a high distinguishing capacity in soft tissues and the possibility of multiparameter comparison and imaging (10). 3D-TOF MRA is the most common angiography technology in clinical practice, and the primary advantage of 3D-TOF MRA is the acquisition of a wide range of cerebral artery MRA images in a relatively short period. The negative results are highly accurate; however, 3D-TOF MRA possesses the disadvantage of exaggerating the extent of cerebrovascular stenosis. In certain cases, lumens with no hemadostenosis may exhibit interrupted imaging or no image development with 3D-TOF MRA.

Black-blood MRA, also known as spatial presaturation MRI, is an alternative MRA technology. Black-blood MRA is able to convert the lumen into a low signal and display the vessel walls with increased image clarity. Therefore, black-blood MRA is able to determine the presence and extent of stenosis, in addition to accurately identifying plaques. Furthermore, by optimizing the TR, TE and NEX parameters, different components of the plaque may be examined. Using black-blood MRA, the type and vulnerability of the plaque are identifiable and the potential risk of cerebral arteriosclerosis may be preliminarily assessed. Thus, black-blood MRA provides an effective approach to the early clinical detection of cerebral infarction (11).

To date, numerous studies have been published on carotid artery HRMRI (12-19), and comparisons between MRI observations of the atherosclerotic plaques in living organisms and pathological specimens have been conducted. Lipid produces a high signal in T1W1, T2W1 and PDWI, while fat saturation is presented as a low signal in T1WI. In addition, fibrous caps are equisignal or produce relatively low signals, and a hemorrhage produces a high signal in T1W1, T1W1 fat saturation, T2W1 and PDWI. For each sequence that occurs, calcification produces a low signal. However, there are limited number of studies that have used MRI to assess the various components of ICAS plaques. ICAS is an integral factor in systematic atherosclerosis. The histological and pathological development of ICAS plaques is similar to that of cervical atherosclerotic plaques. The primary features of the plaques include a fibrous cap, lipid core, calcification and the exudation of inflammatory cells (20). Therefore, the identification of components in ICAS plaques is feasible through comparison with living carotid artery plaque MRI.

In the present study, 110 patients with cerebral infarction were examined using bright-blood 3D-TOF MRA in order to identify the existence of cerebral artery stenosis. Subsequently, black-blood MRA was used for the multisequencing examination of vessels in the abnormal sections. Since the blood vessels in the brain are thinner and exhibit smaller plaques compared with the carotid artery, the scanning vision was reduced and the NEX was increased during MRI scanning. Furthermore, the matrix should be enlarged and the scanning angle adjusted. Ultimately, the expected images were obtained. Bright-blood MRA identified that $16 / 110$ patients possessed an abnormal cerebral artery, with no lumen stenosis, walls or plaques. This result may explain the findings of the clinical bright-blood test. For patients with cerebral artery stenosis or occlusion, no significant ischemia or infarction was identified in the cerebral tissues in the area supplied by the vessel. Therefore, these observations may prevent misdiagnosis and excessive treatment. For the 94 patients with arteriosclerotic plaques identified by black-blood MRA, the revised plaque standard of atherosclerotic plaque classification by Cai et al (2) was adopted in order to observe and analyze the ICAS plaques. Six plaques were categorized as types I-II, in which the wall thickness was approximately normal and four cases exhibited no calcification (Fig. 2). There were 15 plaques classified as type III, which exhibited artery intima diffusion or eccentric thickening (Fig. 3). In total, 26 plaques were classified as types IV-V, and these exhibited a wide range of necrosis with lipid cores and the formation of a fibrous cap in the plaques, which were combined with a small amount of calcification (Fig. 4). A total of 23 cases presented with type VI plaques, in which an anabrosis on the plaque surface, hemorrhage in the plaque or thrombosis was observed (Fig. 5). Type VII plaques were observed in 11 cases, and were defined as calcified plaques (Fig. 6), while type VIII plaques were observed in 14 cases and presented as fibrous plaques without a lipid core, combined with a small amount of calcification (Fig. 7). Finally, one case exhibited a basilar arterial occlusion, with complex plaque components, which was classified as a mixed plaque (Fig. 8). The majority of patients included in the present study presented with unstable ICAS plaques of types IV, V and VI, which may be attributed to the selection of the patient population for the study.

However, there are disadvantages to black-blood MRA in plaque examination, including a long scanning period, no re-establishment of images and a limited examination range of vessels in abnormal sections. Subsequently, the effectiveness of black-blood MRA in clinical practice is limited. Therefore, at present, the combined application of bright- and black-blood MRA is considered to be the most effective approach for identifying cerebrovascular stenosis, the degree of lumen stenosis, the atherosclerotic plaque type and the risks of the plaque. This combined approach may provide a reliable method for the effective individualized clinical treatment of ICAS plaques at an early stage.

\section{References}

1. Park KY, Chung CS, Lee KH, Kim GM, Kim YB and Oh K: Prevalence and risk factors of intracranial atherosclerosis in an asymptomatic korean population. J Clin Neurol 2: 29-33, 2006.

2. Cai JM, Hatsukami TS, Ferguson MS, Small R, Polissar NL and Yuan C: Classification of human carotid atherosclerotic lesions with in vivo multicontrast magnetic resonance imaging. Circulation 106: 1368-1373, 2002.

3. Saam T, Cai JM, Cai YQ, et al: Carotid plaque composition differs between ethno-racial groups: an MRI pilot study comparing mainland Chinese and American Caucasian patients. Arterioscler Thromb Vasc Biol 25: 611-616, 2005.

4. Guntheroth WG: A critical review of the American College of Cardiology/American Heart Association practice guidelines on bicuspid aortic valve with dilated ascending aorta. Am J Cardiol 102: 107-110, 2008 
5. Clarke SE, Hammond RR, Mitchell JR and Rutt BK: Quantitative assessment of carotid plaque composition using multicontrast MRI and registered histology. Magn Reson Med 50: 1199-1208, 2003.

6. Honda M, Kitagawa N, Tsutsumi K, Nagata I, Morikawa M and Hayashi T: High-resolution magnetic resonance imaging for detection of carotid plaques. Neurosurgery 58: 338-346, 2006.

7. Yuan C, Zhang SX, Polissar NL, et al: Identification of fibrous cap rupture with magnetic resonance imaging is highly associated with recent transient ischemic attack or stroke. Circulation 105: 181-185, 2002.

8. Cappendijk VC, Kessels AG, Heeneman S, et al: Comparison of lipid-rich necrotic core size in symptomatic and asymptomatic carotid atherosclerotic plaque: Initial results. J Magn Reson Imaging 27: 1356-1361, 2008.

9. Kantelhardt SR, Greke C, Keric N, Vollmer F, Thiemann I and Giese A: Image guidance for transcranial Doppler ultrasonography. Neurosurgery 68 (Suppl 2): 257-266, 2011.

10. Futami K, Sano H, Misaki K, Nakada M, Ueda F and Hamada J: Identification of the inflow zone of unruptured cerebral aneurysms: comparison of 4D flow MRI and 3D TOF MRA data. AJNR Am J Neuroradiol 35: 1363-1370, 2014.

11. Okuchi S, Okada T, Ihara M, et al: Visualization of lenticulostriate arteries by flow-sensitive black-blood MR angiography on a 1.5 T MRI system: A comparative study between subjects with and without stroke. AJNR Am J Neuroradiol 34: 780-784, 2013.

12. Yuan C, Mitsumori LM, Ferguson MS, et al: In vivo accuracy of multispectral magnetic resonance imaging for identifying lipid-rich necrotic cores and intraplaque hemorrhage in advanced human carotid plaques. Circulation 104: 2051-2056, 2001

13. Yuan C, Miller ZE, Cai J and Hatsukami T: Carotid atherosclerotic wall imaging by MRI. Neuroimaging Clin N Am 12 391-401, 2002.
14. Chiu B, Shamdasani V, Entrekin R, Yuan C and Kerwin WS: Characterization of carotid plaques on 3-dimensional ultrasound imaging by registration with multicontrast magnetic resonance imaging. J Ultrasound Med 31: 1567-1580, 2012.

15. Millon A, Mathevet JL, Boussel L, Faries PL, Fayad ZA, Douek PC and Feugier P: High-resolution magnetic resonance imaging of carotid atherosclerosis identifies vulnerable carotid plaques. J Vasc Surg 57: 1046-1051, 2013.

16. Wang Q, Zeng Y, Wang Y, Cai J, Cai Y, Ma L and Xu X Comparison of carotid arterial morphology and plaque composition between patients with acute coronary syndrome and stable coronary artery disease: A high-resolution magnetic resonance imaging study. Int $\mathbf{J}$ Cardiovasc Imaging 27: 715-726, 2011

17. Chu B, Kampschulte A, Ferguson MS, et al: Hemorrhage in the atherosclerotic carotid plaque: a high-resolution MRI study. Stroke 35: 1079-1084, 2004.

18. Kerwin W, Hooker A, Spilker M, Vicini P, Ferguson M, Hatsukami T and Yuan C: Quantitative magnetic resonance imaging analysis of neovasculature volume in carotid atherosclerotic plaque. Circulation 107: 851-856, 2003.

19. Kerwin WS, O'Brien KD, Ferguson MS, Polissar N, Hatsukami TS and Yuan C: Inflammation in carotid atherosclerotic plaque: a dynamic contrast-enhanced MR imaging study. Radiology 241: 459-468, 2006.

20. Chen XY, Wong KS, Lam WW, Zhao HL and Ng HK: Middle cerebral artery atherosclerosis: histological comparison between plaques associated with and not associated with infarct in a postmortem study. Cerebrovasc Dis 25: 74-80, 2008. 